\title{
Hole transport mechanism in organic/inorganic hybrid system based on in-situ grown cadmium telluride nanocrystals in poly(3-hexylthiophene)
}

\author{
Mohd Taukeer Khan, ${ }^{1,2}$ Amarjeet Kaur, ${ }^{2}$ S. K. Dhawan, ${ }^{1, a)}$ and Suresh Chand ${ }^{1, b)}$ \\ ${ }^{1}$ Polymeric and Soft Materials Section, National Physical Laboratory, (CSIR), Dr. K. S. Krishnan Road, \\ New Delhi-110 012, India \\ ${ }^{2}$ Department of Physics \& Astrophysics, University of Delhi, Delhi-110 007, India
}

(Received 29 March 2011; accepted 21 April 2011; published online 9 June 2011)

\begin{abstract}
The present manuscript demonstrates the hole transport mechanism in an organic/inorganic hybrid system based on in-situ grown cadmium telluride (CdTe) nanocrystals in a poly(3-hexylthiophene) (P3HT) matrix. The increase of hole current in the hybrid system is correlated with the formation of a host-guest (P3HT-CdTe) charge transfer complex duly supported by photoluminescence quenching. The hole transport mechanism in P3HT is governed by a space charge limited current with temperature, carrier density, and field dependent mobility. Incorporation of CdTe nanocrystals in a polymer matrix results in enhancement in the value of trap density $H_{b}$ from $2.8 \times 10^{18}$ to $5.0 \times 10^{18} \mathrm{~cm}^{-3}$ and reduction in activation energies from $52 \mathrm{meV}$ to $11 \mathrm{meV}$. At high trap density, trap potential wells start overlapping; this results in decrease of activation energies.

(C) 2011 American Institute of Physics. [doi:10.1063/1.3594647]
\end{abstract}

\section{INTRODUCTION}

Conjugated polymer poly(3-hexylthiophene) (P3HT) is well known for its application in solar cells as an electron donor material in combination with n-type inorganic nanocrystals, which results in better electron transportation and improved efficiency. ${ }^{1-6}$ In comparison to the all organic electron donor:acceptor devices, this hybrid approach of the photo-active medium is supposed to be the better option as they possess the desirable characteristics of both organic (absorption) and inorganic (transport) systems. Up to now, the organic-inorganic hybrid thin film solar cells have exhibited a power conversion efficiency of up to $\eta \sim 3 \%$, which is relatively small compared to the P3HT:PCBM system $(\eta \sim 6 \%) .{ }^{8}$ The lower $\eta$ in hybrid system is because of an inadequate charge transfer between polymer-nanocrystals and the poor nanoscale morphology of the composites film. In a conventional route, nanocrystals are synthesized through the use of surface ligands. The incorporation of such nanocrystals with the polymer would form an insulating interface, which hinders the electron transfer from the photo-excited polymer to the nanoparticles. ${ }^{9}$ The in-situ growth of the nanocrystals in polymer templates controls the dispersion of the inorganic phase in organic thus providing a large distributed surface area for charge separation. Moreover, nanoparticles are uniformly dispersed in the entire device thickness and thus contain a built in percolation pathway for transport of charge carriers to the respective electrodes.

The main elements used in hybrid solar cells are P3HT (donor) and CdSe nanocrystals (acceptor). As CdTe absorb a higher amount of solar radiation compared to the cadmium Selenide (CdSe), replacement of CdSe with CdTe therefore would enable these hybrid devices to be further enhanced in power conversion efficiency. This new photovoltaic element,

\footnotetext{
a)Electronic mail: skdhawan@mail.nplindia.ernet

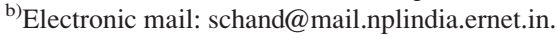

i.e., P3HT incorporated with in-situ grown CdTe nanocrystals, could provide a new nanoscale criterion for the investigation of photoinduced energy/charge transport in organic/ inorganic interfaces. The improved performance of hybrid solar cells is expected because of the improved charge transfer at the organic/inorganic interfaces ${ }^{10}$ and enhanced solar radiation absorption. There are practically no discussions available on P3HT incorporated with CdTe nanocrystals related to the study on its charge transport.

The objective of this paper is to study the effect of in-situ incorporation of CdTe nanocrystals on the hole transport in P3HT conjugated system. For this, we have studied current density versus voltage $(J-V)$ characteristics of pure P3HT as well as P3HT-CdTe nanocomposite thin films in a hole-only device configuration as a function of temperature. Based on these investigations, we have found very interesting and important results wherein the incorporation of CdTe nanocrystals in P3HT matrix results in the enhancement in the hole current and change the transport mechanism from the mobility model to the trap model.

\section{THEORY}

\section{A. Exponential distribution of trap states}

Due to the low mobility of charge carriers in organic semiconductors, the injected carrier forms a space charge. This space charge creates a field that opposes the applied bias and thus decreases the voltage drop across junction; as a result, space charge limited currents (SCLCs) have been proposed as the dominant conduction mechanism in organic semiconductors. ${ }^{11,12}$ Ohmic conduction can be described by

$$
J=q n \mu \frac{V}{d},
$$

where $q$ is the elementary charge, $d$ is the thickness of the film, $\mu$ is the carrier mobility, and $n$ is the carrier density. Pure SCLC with no traps is given by Child's law ${ }^{13}$ 


$$
j=\frac{9}{8} \varepsilon_{r} \varepsilon_{0} \mu \frac{V^{2}}{d^{3}},
$$

where $\varepsilon_{r} \varepsilon_{0}$ is the dielectric permittivity.

SCLC theory with an exponential trap distribution proposes that the space charge that limits conduction is stored in the traps. The exponential distribution of traps in energy and space is described as ${ }^{14}$

$$
N(E)=\frac{H_{b}}{E_{t}} \exp \left(-\frac{E}{E_{t}}\right),
$$

where $N(E)$ is the distribution function of hole trap density at an energy level $E$ above the valence bandedge, $H_{b}$ is the total trap density at the edge of valence band. $E_{t}$ is the characteristic trap energy that is often expressed in terms of the characteristic temperature of trap distribution $T_{C}$ as $E_{t}=k_{B} T_{C}$ and $l=E_{t} / k_{B} T=T_{C} / \mathrm{T}$. where $k_{B}$ is the Boltzmann constant. The parameter $l$ determines the distribution of traps in the forbidden gap.

In case of exponential distribution of traps, assuming that the trapped hole carrier density $\left(p_{t}\right)>>$ free hole carrier density $(p)$ and using continuity equation and boundary condition for current density $(J)$ and applied voltage $(V)$ as

$$
\begin{gathered}
J=q \mu p(x) F(x), \\
V=\int F(x) d x .
\end{gathered}
$$

The expression for $J$ is given by

$$
J=q^{1-l} \mu N_{v}\left(\frac{2 l+1}{l+1}\right)^{l+1}\left(\frac{l}{l+1} \frac{\varepsilon_{r} \varepsilon_{0}}{H_{b}}\right)^{l} \frac{V^{l+1}}{d^{2 l+1}},
$$

where $F(x)$ is the electric field inside the film and $N_{v}$ is the effective density of states. From Eq. (6), the slope of the current-voltage characteristics on a log-log plot is $l+1$. Therefore from the slopes on the log-log plots of current density versus voltage, one can extract the trap energy width $E_{t}$.

\section{B. Unified mobility model}

This model is based on percolation in a variable range hopping (VRH) system with an exponential distribution of localized states. ${ }^{15-17}$ Percolation is the term used for movement of charge carriers through a random network of obstacles. Consider a square lattice, where each site is randomly occupied or empty. Occupied sites are assumed to be electrical conductors while the empty sites represent insulators, and the electrical current can flow between nearest neighbor conductor sites. Percolation paths are the most optimal paths for current and transport of charge carriers, which are governed by the hopping of charge carriers between these conducting sites. The system can be described as a random resistor network, ${ }^{18}$ a system made up of individual disconnected clusters of conducting sites the average size of which is dependent on a reference conductance $G$. The conductance between sites is given by

$$
G=G_{0} \exp \left[-s_{i j}\right]
$$

with

$$
s_{i j}=2 \alpha r_{i j}+\frac{\left|E_{j}-E_{i}\right|+\left|E_{i}-E_{F}\right|+\left|E_{j}-E_{F}\right|}{2 k_{B} T} .
$$

All conductive pathways between sites with $G_{i j}<G$ are electrical insulators, while conductive pathways between sites with $G_{i j} \geq G$ are electrical conductors. At some critical conductance in between, therefore, a threshold conductance $G_{C}$ exists where for the first time, electrical current can percolate from one edge to the other.

A bond is defined as a link between two sites that have a conductance $G_{i j} \geq G$. The average number of bonds $B$ is equal to the density of bonds; $\left(N_{b}\right)$ divided by the density of sites that form bonds, $\left(\boldsymbol{N}_{s}\right)$ in the material. Critical bond number $B_{C}$ is the average number of bonds per site for which threshold percolation occurs. The onset of percolation is determined by calculating the critical average number of bonds per site ${ }^{17}$

$$
B\left(G=G_{C}\right)=B_{C}=\frac{N_{b}}{N_{S}} .
$$

Vissenberg and Matters ${ }^{16,19,20}$ set the critical bond number to $B_{c}=2.8$. The total density of bonds is given by

$$
N_{b}=4 \pi \iiint r_{i j}^{2} g\left(E_{i}\right) g\left(E_{j}\right) \theta\left(s_{c}-s_{i j}\right) d E_{i} d E_{j} d r_{i j} .
$$

The density of sites $N_{s}$

$$
N_{s}=\int g(E) \theta\left(s_{c} k_{B} T-\left|E-E_{F}\right|\right) d E .
$$

At a low carrier concentration, exponential density of states in amorphous organic semiconductors is given by: ${ }^{17,20}$

$$
g(E)=\left\{\begin{array}{l}
\frac{N_{0}}{k_{B} T_{0}} \exp \left[\frac{E}{k_{B} T_{0}}\right], \quad-\infty<E \leq 0, \\
0, E>0
\end{array}\right.
$$

where $N_{o}$ is the total density of states (molecular density) per unit volume and $T_{o}$ is a characteristic temperature that determines the width of the exponential distribution.

Combining Eqs. (8) to (12), the expression for $B_{c}$

$$
B_{C}=\pi N_{0}\left(\frac{T_{0}}{2 \alpha T}\right)^{3} \exp \left[\frac{E_{\max }}{k_{B} T_{0}}\right],
$$

where $E_{\max }=E_{F}+s_{C} k_{B} T$ is the maximum energy that participates in bond formation. According to the percolation theory, the conductivity of the system can be expressed as

$$
\sigma=\sigma_{0} \exp \left[-s_{C}\right]
$$

where $\sigma_{0}$ is the prefactor and $s_{c}$ is the critical exponent of the critical conductance when percolation first occurs (when $B=B_{c}$ ). Using Eqs. (13) and (14), we get

$$
\sigma=\sigma_{0}\left[\left(\frac{T_{0}}{T}\right)^{4} \frac{\sin \left(\pi \frac{T}{T_{0}}\right)}{B_{C}(2 \alpha)^{3}} p\right]^{T_{0} / T} .
$$


The conductivity can be converted into mobility by dividing by $e . p$, where $e$ is the electronic charge and $p$ is the carrier density: ${ }^{21,22}$

$$
\mu(T, p, F)=\frac{\sigma_{0}}{q}\left[\left(\frac{T_{0}}{T}\right)^{4} \frac{\sin \left(\pi \frac{T}{T_{0}}\right)}{B_{C}(2 \alpha)^{3}}\right]^{T_{0} / T} p^{\left(\frac{T_{0}}{T}\right)-1} .
$$

The average charge carrier density as a function of the applied bias voltage $V$ is given by ${ }^{13}$

$$
p(V)=0.75\left[\frac{\varepsilon_{0} \varepsilon_{r} V}{q d^{2}}\right] .
$$

\section{EXPERIMENTAL}

CdTe nanocrystals ( $\sim 5 \mathrm{~nm}$ ) have been grown in-situ in a P3HT matrix. In a typical synthesis of P3HT11, $50 \mathrm{mg}$ $\mathrm{P} 3 \mathrm{HT}$ has been dissolved in $10 \mathrm{ml}$ chlorobenzene. Cadmium acetate dihydrate, $0.2 \mathrm{mmol}(0.1 \mathrm{mmol}$ for P3HT1.5, 0.4 mmol for P3HT12, and $0.6 \mathrm{mmol}$ for P3HT13), was added in $\mathrm{P} 3 \mathrm{HT}$-chlorobenzene solution. The reaction mixture was heated for $2 \mathrm{hr}$ at $160^{\circ} \mathrm{C}$. A tellurium precursor was made by treating $0.4 \mathrm{mmol}(0.2 \mathrm{mmol}$ for P3HT1.5 or $0.8 \mathrm{mmol}$ for P3HT12, and $1.2 \mathrm{mmol}$ for P3HT13) tellurium powder (Acros organics) in trioctylphosphine (TOP) (Sigma Aldrich, USA), at $160^{\circ} \mathrm{C}$ for $2 \mathrm{hr}$ under argon flow. The tellurium precursor so obtained has then injected into the P3HT-Cd solution, and the resultant bright orange reaction mixture was allowed to react for $2 \mathrm{hr}$ at $160{ }^{\circ} \mathrm{C}$ under argon atmosphere. Growth of CdTe NCs has been completed when the solution color turned black. After completion of the reaction, the unreacted $\mathrm{Cd}$-acetate and $\mathrm{Te}$ precursor were been removed by treating nanocomposites with hexane. The reaction mixture was separated by centrifugation and dried in vacuum at $80^{\circ} \mathrm{C}$.

The effect of CdTe nanocrystals on the hole conduction in P3HT has been carried out at different temperatures in hole only device configuration. For this purpose, the hole conduction mechanisms of P3HT as well as P3HT-CdTe nanocomposites thin films have been investigated in device configuration viz. indium tin oxide (ITO)/poly(3,4-ethylendioxythiophene)-poly(styrene sulfonate) (PEDOT:PSS)/ $\mathrm{P} 3 \mathrm{HT} / \mathrm{Au}$ and ITO/PEDOT:PSS/P3HT-CdTe/Au, respectively. These devices are designated as device $\mathrm{A}$ and device $\mathrm{B}$, respectively. Work functions of Au and ITO are close to the highest occupied molecular-orbital (HOMO) of P3HT and CdTe as well as far below the lowest unoccupied molecular-orbital (LUMO) energy level (Fig. 2(a)); as a result, in the Au: ITO device, the transport is dominated by holes. For the preparation of devices A and B, ITO (sheet resistance, $\sim 18 \Omega / \mathrm{cm}^{2}$ ) substrates have been carefully cleaned in ultrasonic baths of acetone and isopropyl alcohol and dried at $120^{\circ} \mathrm{C}$ for $60 \mathrm{~min}$ in vacuum. PEDOT:PSS layers have been spin-coated at $2000 \mathrm{rpm}$ for 2 min onto the ITO substrate and cured at $120^{\circ} \mathrm{C}$ for $60 \mathrm{~min}$ in vacuum. P3HT and P3HTCdTe both have been taken with a concentration of $20 \mathrm{mg} /$ $\mathrm{ml}$ in chlorobenzene. Thin films of P3HT and P3HT-CdTe have been spin casted in inert atmosphere, followed by annealing at $120^{\circ} \mathrm{C}$ for $30 \mathrm{~min}$. Finally, gold $(\mathrm{Au})$ contact, $200 \mathrm{~nm}$, has been applied via evaporation through a shadow mask at $2 \times 10^{-6}$ Torr. The device active areas have been taken $\sim 0.1 \mathrm{~cm}^{2}$ for all the devices discussed in this work. $J-V$ characteristics of the devices have been measured with Keithley 2400 Source-Measure unit, interfaced with a computer.

\section{RESULTS AND DISCUSSION}

Figure 1(a) shows the transmission electron micrograph (TEM) of P3HT-CdTe nanocomposite. Nanocrystals of CdTe have been found in between the polymers chain. The inset of Fig. 1(a) shows the high resolution TEM (HRTEM) and electron diffraction pattern of CdTe nanocrystals in P3HT matrix. In Fig. 1(b), we compared photoluminescence (PL) spectra of pristine P3HT with that of different nanocomposites of P3HT-CdTe. The PL intensity of the nanocomposites has been significantly reduced as compared to the value of pristine P3HT, and the reduction of PL intensity has been increased with the increase of the CdTe concentration in polymer. The reason for the photoluminescence quenching in composites may be due to the $\pi-\pi$ interaction of P3HT with CdTe, ${ }^{23}$ forming additional decaying paths of the excited electrons through the CdTe (see Fig. 2(a)). Reduced PL intensity of the composites relative to the reference P3HT, indicates the host-guest charge transfer complex formation and, thereby, the occurrence of exciton dissociation at interface between P3HT and CdTe-NCs. ${ }^{24}$ This PL quenching experiment provides good evidence that the nanoparticles will be able to transfer their excited state hole to the polymer.

Figure 2 shows the $J-V$ characteristics of device A measured in the temperature range of 290-150 K. On


FIG. 1. (Color online) (a) TEM images of above synthesized CdTe nanocrystals in P3HT matrix (Bar scale $10 \mathrm{~nm}$ ). Inset shows electron diffraction patterns of P3HT-CdTe nanocomposites. (b) Photoluminescence spectra of P3HT and different P3HT-CdTe nanocomposites films after excitation by radiation of $375 \mathrm{~nm}$ wavelengths. 


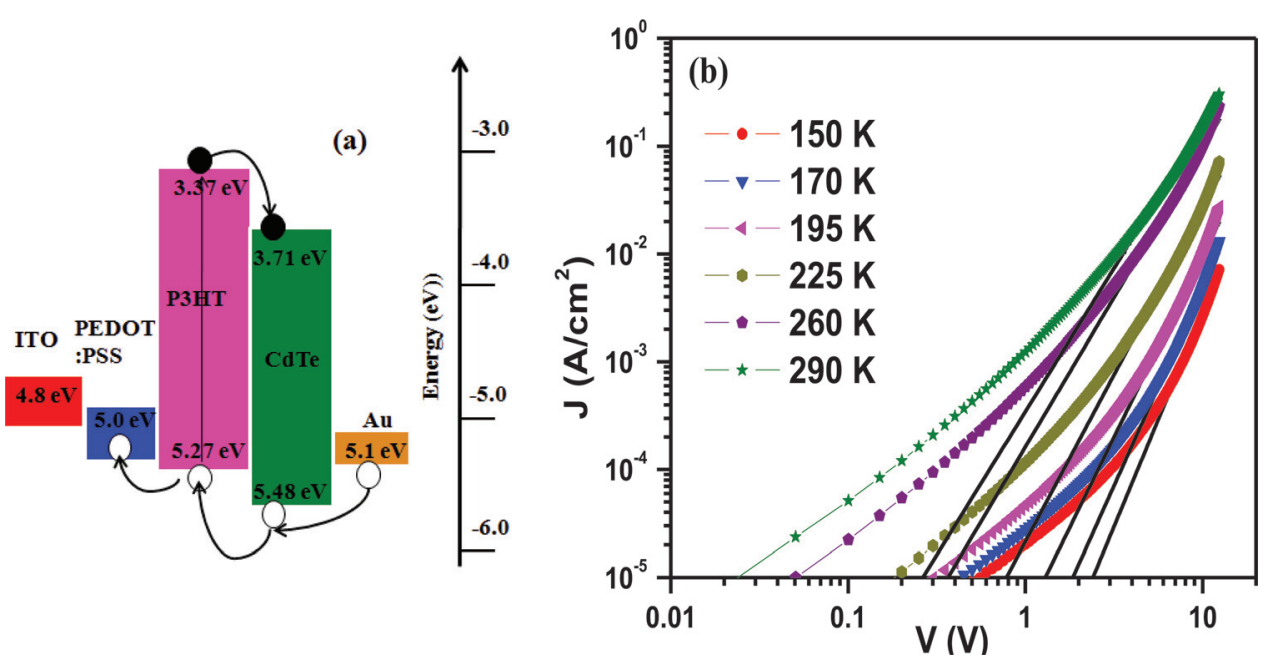

FIG. 2. (Color online) (a) Schematic illustration of the energy diagram of configuration of device B. The P3HT, CdTe have HOMO levels at 5.27, 5.48 while LUMO levels at $3.37,3.71$, respectively for facilitating the charge transfer at the P3HT-CdTe. (b) Experimental (symbols) and calculated (solid lines) $J-V$ characteristic of $\mathrm{P} 3 \mathrm{HT}$ thin films at different temperatures in hole only device configuration viz. ITO/ PEDOT:PSS/P3HT/Au. lowering the temperature, the current has been found to decrease. In organic semiconductors, charge transport is governed by hopping of a carrier from site to site of an empty density of states. The thermal energy helps to cross the energetic barrier between two adjacent sites. This implies that the charge transport in organic semiconductor is thermally activated. Therefore on lowering the temperature, the current has been found to decrease.

At low applied bias, the $J-V$ characteristics showed ohmic behavior [Eq. (1)] as injected carriers are negligible compared to that of the applied bias. ${ }^{25}$ At moderate field, the injected carrier density becomes so high that the field due to the carriers dominates the applied bias. At this point, the $J-V$ characteristics may switch to pure SCLC and follow Eq. (2). On further enhancement of field, the quasi-Fermi level intersects an exponential trap distribution, and characteristics will begin to follow Eq. (6). The hole mobility up to this field is constant and also independent of the hole density. The fit of the $J-V$ characteristics of the P3HT device using the Eq. (6) is poor at high applied bias where current density deviates strongly as expected from Eq. (6). This discrepancy has been analyzed by unified mobility model given by Eq. (16). This model accounts the influence of temperature, carrier density, and applied field on the carrier mobility. ${ }^{17}$ The solid curves in Fig. (2) have been obtained by combining Eqs. (6) and (16) using a computer program. The value of different parameters for solid curves are; $d=110 \mathrm{~nm}, \quad \varepsilon_{\mathrm{r}}=3, \varepsilon_{0}=8.85 \times 10^{-14} \mathrm{~F} / \mathrm{cm}$, $H_{b}=2.8 \times 10^{18} \mathrm{~cm}^{-3}, N_{v}=1 \times 10^{19} \mathrm{~cm}^{-3}, T_{C}=400 \mathrm{~K}$, $T_{0}=325 \mathrm{~K}, \sigma_{0}=4 \times 10^{4} \mathrm{~S} / \mathrm{m}, \alpha^{-1}=1.12 \AA$, and $B_{c}=2.8$.

Figure 3 shows the $J-V$ characteristics of device B measured at different temperatures. Interestingly the nature of P3HT-CdTe composite thin film is different than that of pristine P3HT. In case of composite film, the hole current has been observed to be more than that in pristine P3HT at all temperatures. The inset of Fig. 3 shows the comparison of $J-V$ characteristics of devices A and B at $150 \mathrm{~K}$. The composites exhibited an S-shaped characteristic, and the rate of reduction of current with temperature is low compared to that in pristine P3HT. We tried to fit the experimental data with mobility model. The data did not show agreement with the mobility model for single set of parameter values. On the other hand, the comparison of experimental data with Eq. (6) showed a good agreement with same value of parameters at different temperatures. Solid curves in Fig. (3) represent the plot of Eq. (6) at respective temperatures. The values of parameters used in the calculations are; $H_{b}=5.0 \times 10^{18} \mathrm{~cm}^{-3}$, $N_{v}=6.0 \times 10^{18} \mathrm{~cm}^{-3}, \mu=6.0 \times 10^{-5} \mathrm{~cm}^{2} \mathrm{~V}^{-1} \mathrm{~s}^{-1}, d=110$ $\mathrm{nm}$, and $T_{c}=400 \mathrm{~K}$. For the characteristics measured at 250 $\mathrm{K}, 220 \mathrm{~K}, 195 \mathrm{~K}, 175 \mathrm{~K}$, and $150 \mathrm{~K}$, the agreement was obtained for $\mu=7.8 \times 10^{-5}, 1.16 \times 10^{-4}, 2.4 \times 10^{-4}$, $3.55 \times 10^{-4}, 7.5 \times 10^{-4} \mathrm{~cm}^{2} \mathrm{~V}^{-1} \mathrm{~s}^{-1}$, respectively.

The enhancement in current density in P3HT-CdTe thin film can be understood in terms of host (P3HT) and guest (CdTe) charge transfer type interaction. In fact, there are various possibilities by which CdTe can interact with host P3HT. It can either go structurally into P3HT main chain or form donor/acceptor charge transfer complexes (CTCs) or form molecular aggregates. However, the enhancement in $J$ in device B indicates that CTCs formation between the host

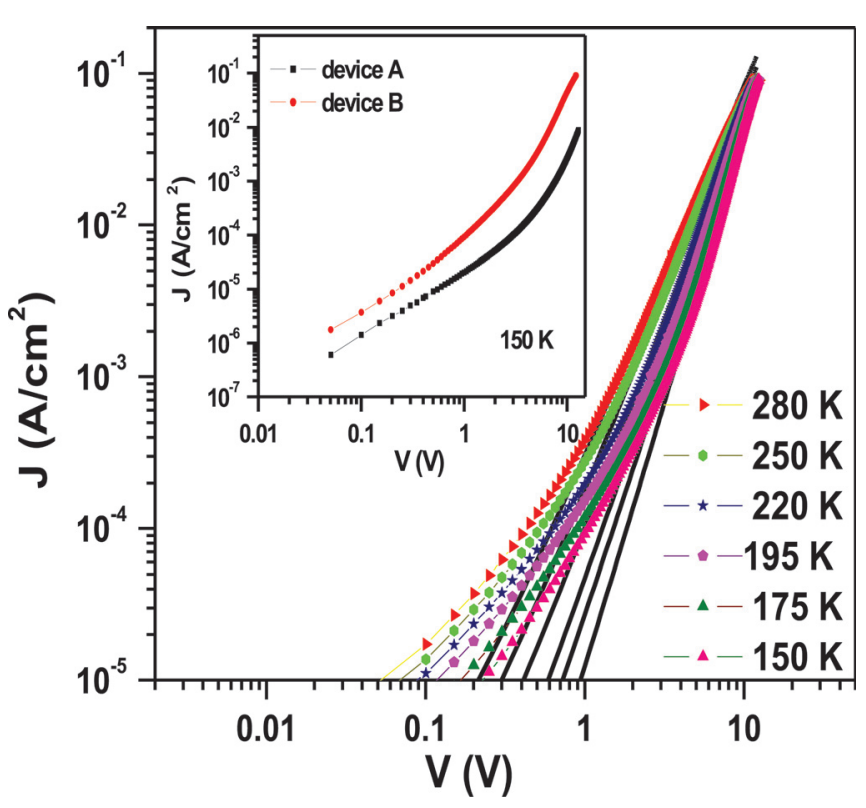

FIG. 3. (Color online) Experimental (symbols) and calculated (solid lines) $J-V$ characteristics of device B at different temperature in hole only device configuration viz. ITO/PEDOT:PSS/P3HT-CdTe/Au. The inset shows the comparison of $J-V$ characteristics of device A and B at $150 \mathrm{~K}$. 
and guest may be the dominant mechanism of interaction between the two, duly supported by photoluminescence quenching [Fig. 1(b)]. In the composites film, the CdTe nanocrystals are bound with P3HT via dipole-dipole interaction and form a CTC. The charge carriers, which had to jump from one chain to another to transport through P3HT, are now assisted by the CdTe nanocrystals. The calculated value of activation energy of localized states has been found to be $52 \mathrm{meV}$ for P3HT and $11 \mathrm{meV}$ for P3HT-CdTe. As activation energy in P3HT-CdTe is lower compared to the pristine $\mathrm{P} 3 \mathrm{HT}$, the CdTe nanocrystals support transportation of holes; this improves their mobility and results into enhancement in the current.

The change of mobility from field dependent in P3HT to field independent in the P3HT-CdTe thin film can be explained on the basis of increase of trap density $\left(H_{b}\right)$ and reduction in activation energy. Usually, an electric field raises the mobility because it lowers the activation barriers. In organic semiconductors, most of the charge carriers are trapped in localized states. An applied field gives rise to the accumulation of charge in the region of the semiconducting layer. As these charges are accumulated, (i) spatial overlap between the trap potential increases that lower the activation barriers ${ }^{26}$ and (ii) only a fraction of total charge carriers are required to fill all the traps, the remaining carriers will on average require less activation energy to hop away to a neighboring site. This results in a higher mobility with increasing field. Incorporation of CdTe nanocrystals in the P3HT matrix simultaneously enhances the value of trap density from $2.8 \times 10^{18}$ to $5.0 \times 10^{18} \mathrm{~cm}^{-3}$ and produces extrinsic charge carriers. ${ }^{27}$ At high trap density, the trap potential wells overlap; this results in decreasing activation energies (from 52 $\mathrm{meV}$ to $11 \mathrm{meV}$ ). Furthermore, increase in the charge carrier density on incorporation of CdTe nanocrystals in P3HT matrix results in only partial filling of carriers even in deeper intrinsic states; this leads to an upward shift of the Fermi level to the effective transport level and concomitant increase of the jump rate. This implies that even at low field larger numbers of free charge carriers are available for transport and hence the mobility in P3HT-CdTe films is independent of applied field.

\section{CONCLUSIONS}

In conclusion, on in-situ incorporation of CdTe-NCs into P3HT matrix, a pronounced change in the transport mechanism and enhancement in hole current of the hybrid system have been observed. Hole transport in pristine P3HT has been observed to follow the unified mobility model, whereas the hybrid film exhibited agreement with the trap conduction mechanism, and mobility is field independent. This change of the conduction mechanism is an important finding and has been attributed to the enhancement in the overlapping of traps potential wells; this results in a decrease in activation energies. Our results constitute important progress for the use of inorganic nanocrystals in polymer for the large area solution processed hybrid photovoltaic cells.

\section{ACKNOWLEDGMENTS}

Authors would like to thank Director NPL for his keen interest in their work. We sincerely thank Dr. Ritu Srivastava, Dr. Rajeev Singh, Dr. Pankaj Kumar, and Dr. Kuldeep Singh, all from NPL, for their cooperation and useful discussions. M.T.K. is thankful to CSIR, India, for the award of Senior Research Fellowship.

${ }^{1}$ M. Reyes-Reyes, K. Kim, and D. L. Carroll, App. Phys. Lett. 87, 083506 (2005).

${ }^{2}$ S. D. Oosterhout, M. M. Wienk, S. S. van Bavel, R. Thiedmann, L. J. A. Koster, J. Gilot, J. Loos, V. Schmidt, and R. A. J. Janssen, Nat. Mater. 8, 818 (2009).

${ }^{3}$ W. Ma, C. Yang, X. Gong, K. Lee, and A. J. Heeger, Adv. Funct. Mater. 15, 1617 (2005).

${ }^{4}$ T. Stoferle, U. Scherf, and R. F. Mahrt, Nano Lett. 9, 453 (2009).

${ }^{5}$ W. U. Huynh, J. J. Dittmer, and A. P. Alivisatos, Science 295, 2425 (2002).

${ }^{6}$ Y. Zhou, Y. Li, H. Zhong, J. Hou, Y. Ding, C. Yang, and Y. Li, Nanotechnology 17, 4041 (2006).

${ }^{7}$ S. Dayal, N. Kopidakis, D. C. Olson, D. S. Ginley, and G. Rumbles, Nano Lett. 10, 239 (2010).

${ }^{8}$ K. Kim, J. Liu, M. A. G. Namboothiry, and D. L. Carroll, Appl. Phys. Lett. 90, 163511 (2007).

${ }^{9}$ W. U. Huynh, J. J. Dittmer, W. C. Libby, G. L. Whiting, and A. P. Alivisatos, Adv. Funct. Mater. 13, 73 (2003).

${ }^{10}$ L. J. A. Koster, W. J. Van Strien, W. J. E. Beek, and P. W. M. Blom, Adv. Funct. Mater. 17, 1297 (2007).

${ }^{11}$ K. C. Kao and W. Hwang, Electrical Transport in Solids (Pergamon, Oxford, 1981)

${ }^{12}$ M. A. Lampert and P. Mark, Current Injection in Solids (Academic, New York, 1970).

${ }^{13}$ P. W. M. Blom and M. C. J. M. Vissenberg, Mater. Sci. Eng. 27, 53 (2000).

${ }^{14}$ M. T. Khan, M. Bajpai, A. Kaur, S.K. Dhawan, and Suresh Chand, Synth. Met. 1601530 (2010)

${ }^{15}$ V. Ambegaokar, B. I. Halperin, and J. S. Langer, Phys. Rev. B 4, 2612 (1971).

${ }^{16} \mathrm{M}$. Sahimi, Applications of Percolation Theory (Taylor and Francis, London, 1994).

${ }^{17}$ F. Torricelli and L. Colalongo, IEEE Electron. Device Lett. 30, 1048 (2009).

${ }^{18}$ H. Scher and E. W. Montroll, Phys. Rev. B 12, 2455 (1975).

${ }^{19}$ G. E. Pike and C. H. Seager, Phys. Rev. B 10, 1421 (1974).

${ }^{20}$ M. C. J. M. Vissenberg and M. Matters, Phys. Rev. B 5712964 (1998).

${ }^{21}$ C. Tanase, E. J. Meijer, P. W. M. Blom, and D. M. de Leeuw, Phys. Rev. Lett. 91, 216601 (2003).

${ }^{22}$ F. Torricelli, D. Zappa, and L. Colalongo, Appl. Phys. Lett. 96, 113304 (2010).

${ }^{23}$ M. T. Khan, R. Bhargav, A. Kaur, S. K. Dhawan, and S. Chand, Thin Solid Films 519, 1007 (2010).

${ }^{24}$ D. S. Ginger and N. C. Greenham, Phys. Rev. B 59, 10622 (1999).

${ }^{25}$ A. J. Campbell, D. D. C. Bradley, and D. G. Lidzey, J. Appl. Phys. 82, 6326 (1997).

${ }^{26}$ P. N. Murgatroyd, J. Phys. D 3, 151 (1970).

${ }^{27}$ V. I. Arkhipov, E. V. Emelianova, P. Heremans, and H. Bässler, Phys. Rev. B 72, 235202 (2005). 